Document downloaded from:

http://hdl.handle.net/10251/39738

This paper must be cited as:

Bonet Solves, JA.; Lindström, M.; Wolf, E. (2012). Norm-attaining weighted composition operators on weighted Banach spaces of analytic functions. Archiv der Mathematik. 99(6):537-546. doi:10.1007/s00013-012-0458-z.

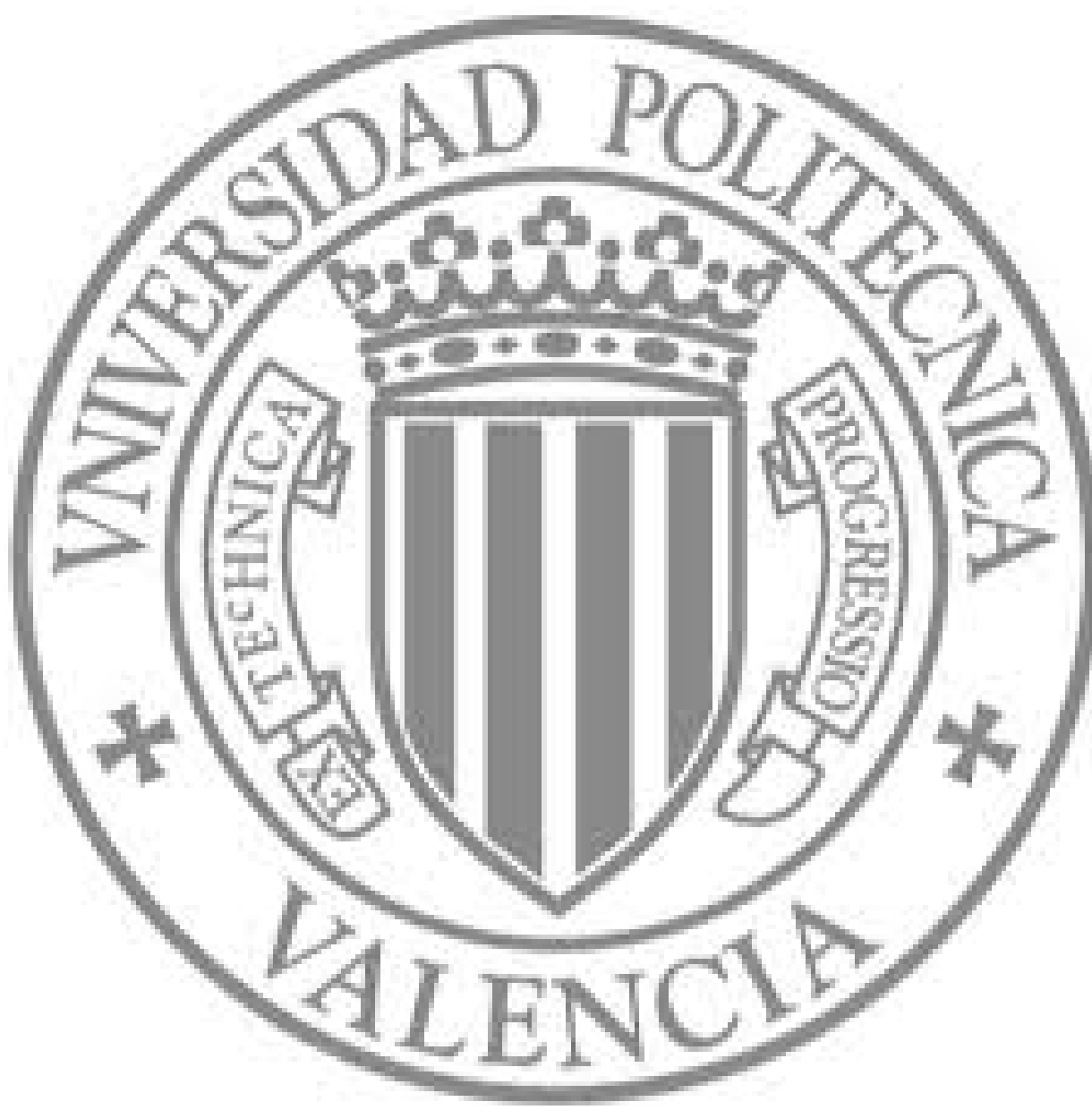

The final publication is available at

http://link.springer.com/article/10.1007\%2Fs00013-012-0458-z

Copyright Springer Verlag (Germany) 


\title{
Norm-attaining weighted composition operators on weighted Banach spaces of analytic functions
}

\author{
José Bonet, Mikael Lindström and Elke Wolf
}

\begin{abstract}
We investigate weighted composition operators that attain their norm on weighted Banach spaces of holomorphic functions on the unit disc of type $H^{\infty}$. Applications for composition operators on weighted Bloch spaces are given.
\end{abstract}

\section{Introduction and Notation}

Let $\phi$ and $\psi$ be analytic maps on the open unit disk $\mathbb{D}$ of the complex plane $\mathbb{C}$ such that $\phi(\mathbb{D}) \subset \mathbb{D}$ and $\psi$ does not coincide with the function zero. These maps induce on the space $H(\mathbb{D})$ of analytic functions on $\mathbb{D}$, via composition and multiplication, a linear weighted composition operator $C_{\phi, \psi}$ defined by $\left(C_{\phi, \psi}\right)(f)=\psi(f \circ \phi)$. Operators of this type have been studied on various spaces of analytic functions. For a discussion of composition operators on classical spaces of analytic functions we refer the reader to the excellent monographs [8] and [16].

Recall that a bounded linear operator $T: X \rightarrow Y$ between Banach spaces $X$ and $Y$ is said to attain its norm on $X$ if there exists $f \in X$ with norm 1 such that $\|T\|=\|T f\|$. We say that a function $f$ with these properties is an extremal function for the norm of $T$. James, see e.g. [9], proved that a Banach space $X$ is reflexive if and only if every compact operator on $X$ is norm-attaining.

Starting with the paper of Hammond [10] in the setting of the Hardy space $H^{2}$, norm-attaining composition operators have been studied on various spaces and by several authors. In particular, Martín [14] characterized norm-attaining composition operators acting on the classical Bloch space $\mathcal{B}$ as well as on the little Bloch space $\mathcal{B}_{0}$. In this note, inspired by her work, we extend her results to the setting of weighted composition operators acting on weighted Banach spaces of holomorphic functions defined below.

A weight $v$ on $\mathbb{D}$ is a strictly positive continuous function on $\mathbb{D}$ which is radial, i.e. $v(z)=$ $v(|z|), z \in \mathbb{D}, v(r)$ is decreasing on $\left[0,1\left[\right.\right.$ and satisfies $\lim _{r \rightarrow 1} v(r)=0$. We associate with a weight $v$ the weighted Banach spaces of holomorphic functions

$$
H_{v}^{\infty}:=\left\{f \in H(\mathbb{D}) ;\|f\|_{v}=\sup _{z \in \mathbb{D}} v(z)|f(z)|<\infty\right\}
$$

and

$$
H_{v}^{0}:=\left\{f \in H_{v}^{\infty} ; \lim _{|z| \rightarrow 1-} v(z)|f(z)|=0\right\},
$$

both endowed with norm $\|\cdot\|_{v}$. The spaces $H_{v}^{\infty}$ and $H_{v}^{0}$ are not reflexive; see [6, 13]. Spaces of this type and composition operators defined on them have been studied thoroughly. See e.g. $[1,3,4,5,7,13,15]$ and the references therein.

2010 Mathematics Subject Classification. Primary: 47H33, Secondary: 46E15, 47B38

Key words and phrases. Weighted composition operators; norm attaining operators; wieghted Banach spaces of holomorphic functions; Bloch spaces.

The research of Bonet was partially supported by MICINN and FEDER Project MTM2010-15200 and by GV project Prometeo/2008/101. 
Our main results are Theorems 2.3 and 3.1. Theorem 2.3 asserts that every bounded weighted composition operator on $H_{v}^{\infty}$ is norm attaining, whereas Theorem 3.1 characterizes the bounded weighted composition operators on $H_{v}^{0}$ which are norm attaining. Examples are provided. Our last section includes consequences for norm attaining composition operators on weighted Bloch spaces.

Here are examples of weights $v(z)$ on $\mathbb{D}$ :

(1) The polynomial weights $v(z)=\left(1-|z|^{2}\right)^{\alpha}, \alpha>0$.

(2) The exponential weights $v(z)=\exp \left(-\frac{1}{(1-|z|)^{\alpha}}\right), \alpha>0$.

(3) The logarithmic weights $v(z)=\left(\log \frac{e}{1-r}\right)^{-\alpha}, \alpha>0$.

The so called associated weight (see [2]) is an important tool to study operators on weighted Banach spaces of analytic functions. For a weight $v$, the associated weight $\tilde{v}$ is defined by

$$
\tilde{v}(z):=\left(\sup \left\{|f(z)| ; f \in H_{v}^{\infty},\|f\|_{v} \leq 1\right\}\right)^{-1}=\left(\left\|\delta_{z}\right\|_{v}\right)^{-1}, z \in \mathbb{D},
$$

where $\delta_{z}$ denotes the point evaluation of z. By [2, Properties 1.2] we know that the associated weight is continuous, radial, $\tilde{v} \geq v>0$ and that for each $z \in \mathbb{D}$ we can find $f_{z} \in H_{v}^{\infty},\left\|f_{z}\right\|_{v}=1$, such that $\left|f_{z}(z)\right| \tilde{v}(z)=1$. It is also shown in [2, Observation 1.12] that $H_{\tilde{v}}^{\infty}$ coincides isometrically with $H_{v}^{\infty}$. In particular, $\|\cdot\|_{v}=\|\cdot\|_{\tilde{v}}$. Under the present assumptions on the weights, it is also true that $H_{\tilde{v}}^{0}$ coincides isometrically with $H_{v}^{0}$; see [4].

The norm of a bounded weighted composition operator $C_{\phi, \psi}: H_{v}^{\infty} \rightarrow H_{w}^{\infty}$, is given by

$$
\left\|C_{\phi, \psi}\right\|_{H_{v}^{\infty} \rightarrow H_{w}^{\infty}}=\sup _{z \in D} \frac{w(z)|\psi(z)|}{\tilde{v}(\phi(z))} .
$$

See $[4,7,15]$. Moreover, by $[7,15]$, the essential norm (i.e. the distance to the compact operators) of a bounded composition operator $C_{\phi, \psi}: H_{v}^{0} \rightarrow H_{w}^{0}$ is given by

$$
\left\|C_{\phi, \psi}\right\|_{e, H_{v}^{0} \rightarrow H_{w}^{0}}=\limsup _{|z| \rightarrow 1-} \frac{w(z)|\psi(z)|}{\tilde{v}(\phi(z))} .
$$

\section{Norm attaining weighted composition operators on $H_{v}^{\infty}$}

The following useful Lemma is due to Horokawa, Izuchi and Zheng [11].

Lemma 2.1 ([11]) Let $\left(z_{m}\right)_{m} \subset \mathbb{D}$ be a sequence such that $\left|z_{m}\right| \rightarrow 1$, when $m \rightarrow \infty$. Then there is a subsequence $\left(z_{n}\right)_{n}$ of $\left(z_{m}\right)_{m}$ and there is a sequence $\left(g_{k}\right)_{k}$ in the disc algebra $A(\mathbb{D})$ such that

$$
\text { (i) } \sup _{z \in \mathbb{D}} \sum_{k=1}^{\infty}\left|g_{k}(z)\right| \leq 1 \text {, }
$$

and

$$
\text { (ii) }\left|g_{n}\left(z_{n}\right)\right|>1-\left(\frac{1}{2}\right)^{n} \text { for all } n \in \mathbb{N} \text {. }
$$

Proof. The statement follows from the proof of Theorem 3.1 in [11]. In the notation of [11], $g_{k}:=c_{k} f^{m_{k}} g_{n_{k}}$ and the subsequences are selected by (3.3)-(3.6) in that paper.

Observe that in the notation of Lemma 2.1, we have $\sum_{k=1, k \neq n}^{\infty}\left|g_{k}\left(z_{n}\right)\right| \leq \frac{1}{2^{n}}$ for each $n \in \mathbb{N}$.

Lemma 2.2 Let $v$ be a weight on $\mathbb{D}$. If $\left(z_{m}\right)_{m} \subset \mathbb{D}$ be a sequence such that $\left|z_{m}\right| \rightarrow 1$, when $m \rightarrow \infty$, then there is a subsequence $\left(z_{n}\right)_{n}$ of $\left(z_{m}\right)_{m}$ and there is $g \in H_{v}^{\infty},\|g\|_{v} \leq 1$, such that $\left|g\left(z_{n}\right)\right| \tilde{v}\left(z_{n}\right) \rightarrow 1$, when $n \rightarrow \infty$. 
Proof. We apply Lemma 2.1 to construct functions $g_{k}$ in the disc algebra $A(\mathbb{D})$ satisfying conditions (i) and (ii). Now, for every $k$, find $f_{k} \in H_{v}^{\infty},\left\|f_{k}\right\|_{v}=1$, such that $f_{k}\left(z_{k}\right) \tilde{v}\left(z_{k}\right)=1$ and put $g(z):=\sum_{k=1}^{\infty} g_{k}(z) f_{k}(z)$. It is easy to see that $g \in H(\mathbb{D})$ and $|g(z)| v(z) \leq 1$ for all $z \in \mathbb{D}$, so $g \in H_{v}^{\infty}$ and $\|g\|_{v} \leq 1$. Moreover, for all $n$,

$$
\begin{gathered}
\left|g\left(z_{n}\right)\right| \tilde{v}\left(z_{n}\right)=\left|\sum_{k=1}^{\infty} g_{k}\left(z_{n}\right) f_{k}\left(z_{n}\right) \tilde{v}\left(z_{n}\right)\right|=\left|g_{n}\left(z_{n}\right) f_{n}\left(z_{n}\right) \tilde{v}\left(z_{n}\right)+\sum_{k=1, k \neq n}^{\infty} g_{k}\left(z_{n}\right) f_{k}\left(z_{n}\right) \tilde{v}\left(z_{n}\right)\right| \\
\geq\left|g_{n}\left(z_{n}\right)\right|-\sum_{k=1, k \neq n}^{\infty}\left|g_{k}\left(z_{n}\right)\right| \geq 1-\left(\frac{1}{2}\right)^{n}-\left(\frac{1}{2}\right)^{n} .
\end{gathered}
$$

Hence $1-\frac{1}{2^{n-1}} \leq\left|g\left(z_{n}\right)\right| \tilde{v}\left(z_{n}\right) \leq 1$ for each $n \in \mathbb{N}$. This implies the conclusion.

Theorem 2.3 (a) Every bounded composition operator $C_{\phi, \psi}: H_{v}^{\infty} \rightarrow H_{w}^{\infty}$ is norm attaining.

(b) A function $f \in H_{v}^{\infty}$ is extremal for $C_{\phi, \psi}: H_{v}^{\infty} \rightarrow H_{w}^{\infty}$ if and only if there is a sequence $\left(z_{n}\right)_{n}$ in $\mathbb{D}$ such that $\lim _{n \rightarrow \infty} \tilde{v}\left(\phi\left(z_{n}\right)\right)\left|f\left(\phi\left(z_{n}\right)\right)\right|=1$ and $\lim _{n \rightarrow \infty} \frac{\left|\psi\left(z_{n}\right)\right| w\left(z_{n}\right)}{\tilde{v}\left(\phi\left(z_{n}\right)\right)}=\left\|C_{\phi, \psi}\right\|$.

Proof. (a) Since the norm of a bounded weighted composition operator $C_{\phi, \psi}: H_{v}^{\infty} \rightarrow H_{w}^{\infty}$, is given by $\left\|C_{\phi, \psi}\right\|=\sup _{z \in D} \frac{w(z)|\psi(z)|}{\tilde{v}(\phi(z))}$, we can find a sequence $\left(z_{n}\right) \subset \mathbb{D}$ and $b \in \overline{\mathbb{D}}$ with $\phi\left(z_{m}\right) \rightarrow b$ as $m \rightarrow \infty$ such that

$$
\lim _{m} \frac{\left|\psi\left(z_{m}\right)\right| w\left(z_{m}\right)}{\tilde{v}\left(\phi\left(z_{m}\right)\right)}=\left\|C_{\phi, \psi}\right\| .
$$

We distinguish two cases.

Case 1: $|b|=1$. We apply Lemma 2.2 to $\left(\phi\left(z_{m}\right)\right)_{m}$ to find a subsequence $\left(z_{n}\right)_{n}$ of $\left(z_{m}\right)_{m}$ and $g \in H_{v}^{\infty},\|g\|_{v} \leq 1$, such that $\lim _{n \rightarrow \infty} g\left(\phi\left(z_{n}\right)\right) \tilde{v}\left(\phi\left(z_{n}\right)\right)=1$.

Case 2: $|b|<1$. There exists a $g \in H_{v}^{\infty},\|g\|_{v}=1$, with $g(b) \tilde{v}(b)=1$. Therefore

$$
\lim _{n \rightarrow \infty} g\left(\phi\left(z_{n}\right)\right) \tilde{v}\left(\phi\left(z_{n}\right)\right)=g(b) \tilde{v}(b)=1 .
$$

We have, in both cases,

$$
\begin{gathered}
\left\|C_{\phi, \psi} g\right\|=\sup _{z \in \mathbb{D}}|\psi(z) \| g(\phi(z))| w(z) \geq \lim _{n} \frac{\left|\psi\left(z_{n}\right)\right|\left|g\left(\phi\left(z_{n}\right)\right)\right| \tilde{v}\left(\phi\left(z_{n}\right)\right) w\left(z_{n}\right)}{\tilde{v}\left(\phi\left(z_{n}\right)\right)}= \\
=\lim _{n} \frac{\left|\psi\left(z_{n}\right)\right| w\left(z_{n}\right)}{\tilde{v}\left(\phi\left(z_{n}\right)\right)}=\left\|C_{\phi, \psi}\right\| .
\end{gathered}
$$

This implies that $g \in H_{v}^{\infty},\|g\|_{v} \leq 1$, is an extremal function for $C_{\phi, \psi}: H_{v}^{\infty} \rightarrow H_{w}^{\infty}$.

(b) The proof of part (a) shows that if $f \in H_{v}^{\infty},\|f\|_{v} \leq 1$, satisfies that there is a sequence $\left(z_{n}\right)_{n}$ in $\mathbb{D}$ such that $\lim _{n \rightarrow \infty} \tilde{v}\left(\phi\left(z_{n}\right)\right)\left|f\left(\phi\left(z_{n}\right)\right)\right|=1$ and $\lim _{n} \frac{\left|\psi\left(z_{n}\right)\right| w\left(z_{n}\right)}{\tilde{v}\left(\phi\left(z_{n}\right)\right)}=|| C_{\phi, \psi} \|$, then $f$ is an extremal function for $C_{\phi, \psi}: H_{v}^{\infty} \rightarrow H_{w}^{\infty}$.

Conversely, assume that $f \in H_{v}^{\infty},\|f\|_{v} \leq 1$, satisfies

$$
\left\|C_{\phi, \psi}\right\|=\left\|C_{\phi, \psi} f\right\|_{w}=\sup _{z \in \mathbb{D}} w(z)|\psi(z)||f(\phi(z))| .
$$

Select a sequence $\left(z_{n}\right)_{n}$ in $\mathbb{D}$ such that, for each $n \in \mathbb{N}$,

$$
\left(1-\frac{1}{n}\right)\left\|C_{\phi, \psi}\right\|<w\left(z_{n}\right)\left|\psi\left(z_{n}\right)\right|\left|f\left(\phi\left(z_{n}\right)\right)\right| \leq\left\|C_{\phi, \psi}\right\| .
$$

Hence, for each $n \in \mathbb{N}$,

$$
\left(1-\frac{1}{n}\right) \frac{w\left(z_{n}\right)\left|\psi\left(z_{n}\right)\right|}{\tilde{v}\left(\phi\left(z_{n}\right)\right)}<w\left(z_{n}\right)\left|\psi\left(z_{n}\right)\right|\left|f\left(\phi\left(z_{n}\right)\right)\right| .
$$


This implies $1-\frac{1}{n}<\tilde{v}\left(\phi\left(z_{n}\right)\right)\left|f\left(\phi\left(z_{n}\right)\right)\right| \leq 1$; the last inequality because $\|f\|_{v}=1$. We get $\lim _{n \rightarrow \infty} \tilde{v}\left(\phi\left(z_{n}\right)\right)\left|f\left(\phi\left(z_{n}\right)\right)\right|=1$. By the inequality above for $\left\|C_{\phi, \psi}\right\|$, we conclude

$$
\lim _{n} \frac{\left|\psi\left(z_{n}\right)\right| w\left(z_{n}\right)}{\tilde{v}\left(\phi\left(z_{n}\right)\right)}=\left\|C_{\phi, \psi}\right\| .
$$

\section{Norm attaining weighted composition operators on $H_{v}^{0}$}

Theorem 3.1 A bounded composition operator $C_{\phi, \psi}: H_{v}^{0} \rightarrow H_{w}^{0}$ is norm attaining if and only if there are $b \in \mathbb{D}$ and $\left(z_{n}\right)_{n}$ in $\mathbb{D}$ with $\lim _{n \rightarrow \infty} \phi\left(z_{n}\right)=b$ such that

$$
\left\|C_{\phi, \psi}\right\|=\lim _{n \rightarrow \infty} \frac{w\left(z_{n}\right)\left|\psi\left(z_{n}\right)\right|}{\tilde{v}\left(\phi\left(z_{n}\right)\right)}
$$

and there is $f \in H_{v}^{0},\|f\|_{v}=1$, (which is an extremal function) satisfying $\tilde{v}(b) f(b)=1$.

Proof. Assume first that $C_{\phi, \psi}: H_{v}^{0} \rightarrow H_{w}^{0}$ is norm attaining. There is $f \in H_{v}^{0},\|f\|_{v}=1$, such that $\left\|C_{\phi, \psi}\right\|=\left\|C_{\phi, \psi} f\right\|_{w}$. Since $f \in H_{v}^{0}=H_{\tilde{v}}^{0}$, there is $\left.R \in\right] 0,1[$ such that $\tilde{v}(\zeta)|f(\zeta)|<1 / 2$ for each $\zeta \in \mathbb{D},|\zeta|>R$. If $z \in \mathbb{D}$ satisfies $|\phi(z)|>R$, then

$$
w(z)|\psi(z)||f(\phi(z))| \leq \frac{1}{2} \frac{w(z)|\psi(z)|}{\tilde{v}(\phi(z))} \leq \frac{1}{2}\left\|C_{\phi, \psi}\right\| .
$$

Set $A:=\{z \in \mathbb{D}|| \phi(z) \mid \leq R\}$. We have

$$
\left\|C_{\phi, \psi}\right\|=\left\|C_{\phi, \psi} f\right\|_{w}=\sup _{z \in A} w(z)|\psi(z)||f(\phi(z))| .
$$

For each $n \in \mathbb{N}$ we select $z_{n} \in A$ with

$$
\left(1-\frac{1}{n}\right)\left\|C_{\phi, \psi}\right\|<w\left(z_{n}\right)\left|\psi\left(z_{n}\right)\right|\left|f\left(\phi\left(z_{n}\right)\right)\right| \leq\left\|C_{\phi, \psi} f\right\|_{w}=\left\|C_{\phi, \psi}\right\| .
$$

Passing to a subsequence if necessary, we may assume that $\lim _{n \rightarrow \infty} \phi\left(z_{n}\right)=b$, with $|b| \leq R<1$, hence $b \in \mathbb{D}$. Moreover, for each $n \in \mathbb{N}$,

$$
\left(1-\frac{1}{n}\right) \frac{w\left(z_{n}\right)\left|\psi\left(z_{n}\right)\right|}{\tilde{v}\left(\phi\left(z_{n}\right)\right)}<w\left(z_{n}\right)\left|\psi\left(z_{n}\right)\right|\left|f\left(\phi\left(z_{n}\right)\right)\right| .
$$

Thus, for each $n \in \mathbb{N}$,

$$
1-\frac{1}{n}<\tilde{v}\left(\phi\left(z_{n}\right)\right)\left|f\left(\phi\left(z_{n}\right)\right)\right| \leq\|f\|_{v}=1 .
$$

This implies $\tilde{v}(b) f(b)=1=\lim _{n \rightarrow \infty} \tilde{v}\left(\phi\left(z_{n}\right)\right)\left|f\left(\phi\left(z_{n}\right)\right)\right|$. From where it follows

$$
\begin{gathered}
|| C_{\phi, \psi}||=\lim _{n \rightarrow \infty} w\left(z_{n}\right)\left|\psi\left(z_{n}\right)\right|\left|f\left(\phi\left(z_{n}\right)\right)\right|= \\
=\lim _{n \rightarrow \infty} \frac{w\left(z_{n}\right)\left|\psi\left(z_{n}\right)\right|}{\tilde{v}\left(\phi\left(z_{n}\right)\right)} \tilde{v}\left(\phi\left(z_{n}\right)\right)\left|f\left(\phi\left(z_{n}\right)\right)\right|=\lim _{n \rightarrow \infty} \frac{w\left(z_{n}\right)\left|\psi\left(z_{n}\right)\right|}{\tilde{v}\left(\phi\left(z_{n}\right)\right)} .
\end{gathered}
$$

The proof of this implication is complete.

To prove the other implication, select $b \in \mathbb{D},\left(z_{n}\right)_{n}$ and $f \in H_{v}^{0},\|f\|_{v}=1$, as in the assumption. We have

$$
\begin{gathered}
\left\|C_{\phi, \psi}\right\|=\lim _{n \rightarrow \infty} \frac{w\left(z_{n}\right)\left|\psi\left(z_{n}\right)\right|}{\tilde{v}\left(\phi\left(z_{n}\right)\right)}=\lim _{n \rightarrow \infty} w\left(z_{n}\right)\left|\psi\left(z_{n}\right)\right|\left|f\left(\phi\left(z_{n}\right)\right)\right| \frac{\tilde{v}(b)}{\tilde{v}\left(\phi\left(z_{n}\right)\right)} \frac{|f(b)|}{f\left(\phi\left(z_{n}\right)\right) \mid}= \\
\lim _{n \rightarrow \infty} w\left(z_{n}\right)\left|\psi\left(z_{n}\right)\right|\left|f\left(\phi\left(z_{n}\right)\right)\right| \leq\left\|C_{\phi, \psi} f\right\|_{w} .
\end{gathered}
$$

This implies that $f \in H_{v}^{0}$ is an extremal function for the operator $C_{\phi, \psi}: H_{v}^{0} \rightarrow H_{w}^{0}$. 
Corollary 3.2 Let $v$ be a weight such that for each $b \in \mathbb{D}$ there is $f \in H_{v}^{0},\|f\|_{v}=1$, such that $\tilde{v}(b) f(b)=1$. If $C_{\phi, \psi}: H_{v}^{0} \rightarrow H_{w}^{0}$ is a bounded operator such that $\left\|C_{\phi, \psi}\right\|_{e}<\left\|C_{\phi, \psi}\right\|$ (for example if $C_{\phi, \psi}$ is compact and non-zero), then $C_{\phi, \psi}: H_{v}^{0} \rightarrow H_{w}^{0}$ is norm attaining.

Proof. According to [15, Theorem 2.2], as $\left\|C_{\phi, \psi}\right\|_{e}<\left\|C_{\phi, \psi}\right\|$,

$$
\limsup _{|z| \rightarrow 1-} \frac{w(z)|\psi(z)|}{\tilde{v}(\phi(z))}<\sup _{z \in \mathbb{D}} \frac{w(z)|\psi(z)|}{\tilde{v}(\phi(z))} .
$$

Select $\left(z_{m}\right)_{m}$ in $\mathbb{D}$ such that $\left\|C_{\phi, \psi}\right\|=\lim _{n \rightarrow \infty} \frac{w\left(z_{n}\right)\left|\psi\left(z_{n}\right)\right|}{\tilde{v}\left(\phi\left(z_{n}\right)\right)}$. By the inequality above, there is a subsequence $\left(z_{n}\right)_{n}$ of $\left(z_{m}\right)_{m}$ such that $z_{n} \rightarrow a \in \mathbb{D}$ as $n \rightarrow \infty$. Put $b=\phi(a)$. By assumption, there is $f \in H_{v}^{0},\|f\|_{v}=1$, such that $\tilde{v}(b) f(b)=1$. We can apply Theorem 3.1 to conclude that $f \in H_{v}^{0}$ is an extremal function for $C_{\phi, \psi}: H_{v}^{0} \rightarrow H_{w}^{0}$.

We discuss now examples of weights satisfying the assumption of Corollary 3.2 :

$$
(* *) \quad \forall b \in \mathbb{D} \quad \exists f \in H_{v}^{0},\|f\|_{v}=1 \text {, such that } \tilde{v}(b) f(b)=1 .
$$

First recall the following notation: For $a \in \mathbb{D}$, we denote by $\sigma_{a}(z):=\frac{a-z}{1-\bar{a} z}$. We have $\sigma_{a}^{\prime}(z)=$ $\frac{|a|^{2}-1}{(1-\bar{a} z)^{2}}$ and $\left|\sigma_{a}^{\prime}(z)\right|=\frac{1-\left|\sigma_{a}(z)\right|^{2}}{1-|z|^{2}}$ for each $z, a \in \mathbb{D}$. See e.g. [17].

Lemma 3.3 The polynomial weights $v(z)=\left(1-|z|^{2}\right)^{\alpha}, \alpha>0$, satisfy the condition (**).

Proof. It is known, see [2], that the polynomial weights satisfy $v=\tilde{v}$. Given $b \in \mathbb{D}$, consider the function

$$
f_{b}(z):=\left(\sigma_{b}^{\prime}(z)\right)^{\alpha}=\left(\frac{|b|^{2}-1}{(1-\bar{b} z)^{2}}\right)^{\alpha} .
$$

Since $f_{b} \in H^{\infty}$, we have $f_{b} \in H_{v}^{0}$. Moreover

$$
\left\|f_{b}\right\|_{v}=\sup _{z \in \mathbb{D}}\left(1-|z|^{2}\right)^{\alpha}\left|\sigma_{b}^{\prime}(z)\right|^{\alpha}=\sup _{z \in \mathbb{D}}\left(1-\left|\sigma_{a}(z)\right|^{2}\right)^{\alpha}=1
$$

Clearly $v(b)\left|f_{b}(b)\right|=1$.

Corollary 3.4 Let $v$ be a polynomial weight $v(z)=\left(1-|z|^{2}\right)^{\alpha}, \alpha>0$, and let $w$ be an arbitrary weight. A bounded composition operator $C_{\phi, \psi}: H_{v}^{0} \rightarrow H_{w}^{0}$ is norm attaining if and only if there are $b \in \mathbb{D}$ and $\left(z_{n}\right)_{n}$ in $\mathbb{D}$ with $\lim _{n \rightarrow \infty} \phi\left(z_{n}\right)=b$ such that

$$
\left\|C_{\phi, \psi}\right\|=\lim _{n \rightarrow \infty} \frac{w\left(z_{n}\right)\left|\psi\left(z_{n}\right)\right|}{\tilde{v}\left(\phi\left(z_{n}\right)\right)} .
$$

Proof. This is a direct consequence of Theorem 3.1 and Lemma 3.3.

Our next proposition permits us to exhibit more examples of weights satisfying condition $(* *)$.

Proposition 3.5 Let $v$ be a weight such that $v=\tilde{v}$ satisfying condition $(* *)$. If $w$ is another weight such that $w=\tilde{w}$, then $u:=v w$ satisfies also the condition.

Proof. First of all it is easy to conclude from [2, Properties 1.2 (iv)] that $\tilde{u}(z) \leq \tilde{v}(z) \tilde{w}(z)$ for each $z \in \mathbb{D}$. This implies $u \leq \tilde{u} \leq \tilde{v} \tilde{w}=v w=u$. Thus $u=\tilde{u}=\tilde{v} \tilde{w}$. Now, to check condition (**) for $u$, fix $b \in \mathbb{D}$. We find $g \in H_{w}^{\infty},\|g\|_{w}=1$ with $g(b)=1 / \tilde{w}(b)=1 / w(b)$. Since $v$ satisfies condition $(* *)$, there is $f \in H_{v}^{0},\|f\|_{v}=1$ such that $f(b)=1 / \tilde{v}(b)=1 / v(b)$. Setting $h=f g$, we have $\|h\|_{u} \leq 1$, $\tilde{u}(b) h(b)=u(b) h(b)=1$ and $h \in H_{u}^{0}$, as $\lim _{|z| \rightarrow 1-} u(z)|h(z)| \leq \lim _{|z| \rightarrow 1-} v(z)|f(z)|=0$. $(* *)$ :

As a consequence of Lemma 3.3 and Proposition 3.5, the following weights satisfy condition 
(1) $u(z)=\left(1-|z|^{2}\right)^{\alpha} \exp \left(-\frac{1}{(1-|z|)^{\beta}}\right), \alpha, b>0$.

(2) $u(z)=\left(1-|z|^{2}\right)^{\alpha}\left(\log \frac{e}{1-r}\right)^{-\beta}, \alpha, b>0$.

(3) $u(z)=1-|z|$. Just take $v(z)=1-|z|^{2}, w(z)=1 /(1+|z|)$ and apply [2, Corollary 1.6].

Example 3.6 Let $v$ be a polynomial weight $v(z)=\left(1-|z|^{2}\right)^{\alpha}, \alpha>0$. Consider the holomorphic self map $\phi(z)=\sigma_{a}(z)$ on $\mathbb{D}$ for some $a \in \mathbb{D}$. The composition operator $C_{\phi}: H_{v}^{0} \rightarrow H_{v}^{0}$ is not norm attaining. Indeed, for each $z \in \mathbb{D}$, we have

$$
\frac{v(z)}{v(\phi(z))}=\frac{\left(1-|z|^{2}\right)^{\alpha}}{\left(1-\left|\sigma_{a}(z)\right|^{2}\right)^{\alpha}}=\frac{1}{\left|\left(\sigma_{a}^{\prime}(z)\right)^{\alpha}\right|}=\frac{|1-\bar{a} z|^{2 \alpha}}{\left(1-|a|^{2}\right)^{\alpha}} .
$$

Accordingly, a sequence $\left(z_{n}\right)_{n}$ in $\mathbb{D}$ with $\left\|C_{\phi}\right\|=\sup _{z \in \mathbb{D}} \frac{v(z)}{v(\phi(z))}=\lim _{n \rightarrow \infty} \frac{v\left(z_{n}\right)}{v\left(\phi\left(z_{n}\right)\right)}$ must satisfy $\left|z_{n}\right| \rightarrow 1$ as $n \rightarrow \infty$. We can apply Corollary 3.4 for $\psi(z)=1$ to conclude that $C_{\phi}: H_{v}^{0} \rightarrow H_{v}^{0}$ is not norm attaining.

Examples 3 and 4 in Martín [14] (see also [12]) have the following consequences in our setting that are relevant in connection with Corollary 3.2 .

Examples 3.7 (1) Let $v$ be a polynomial weight $v(z)=\left(1-|z|^{2}\right)^{\alpha}, \alpha>0$. Consider the holomorphic maps $\phi(z)=(z+1) / 2$ and $\psi(z)=1 / 2$. The weighted composition operator $C_{\phi, \psi}: H_{v}^{0} \rightarrow H_{v}^{0}$ is not norm attaining by Corollary 3.4 and $\left\|C_{\phi, \psi}\right\|_{e}=\left\|C_{\phi, \psi}\right\|$.

(2) Let $v$ be the weight $v(z)=1-|z|^{2}$. Consider the lens map $\phi(z)=\left(\sigma(z)^{\alpha}-1\right) /\left(\sigma(z)^{\alpha}+1\right), 0<$ $\alpha<1$, with $\sigma(z)=(1+z) /(1-z)$, and $\psi(z)=\phi^{\prime}(z)$. The weighted composition operator $C_{\phi, \psi}: H_{v}^{0} \rightarrow H_{v}^{0}$ is norm attaining by Corollary 3.4, but $\left\|C_{\phi, \psi}\right\|_{e}=\left\|C_{\phi, \psi}\right\|$. Thus the converse of Corollary 3.2 does not hold in general.

\section{Consequences for composition operators on weighted Bloch spaces}

Let $v$ be a weight. The weighted Bloch space is defined by

$$
\mathcal{B}_{v}=\left\{f \in H(\mathbb{D}): f(0)=0,\|f\|_{\mathcal{B}_{v}}=\sup _{z \in D} v(z)\left|f^{\prime}(z)\right|<\infty\right\},
$$

and the little Bloch space

$$
\mathcal{B}_{v, 0}=\left\{f \in \mathcal{B}: \lim _{|z| \rightarrow 1-} v(z)\left|f^{\prime}(z)\right|=0\right\} .
$$

They are Banach spaces endowed with the norm $\|\cdot\|_{\mathcal{B}_{v}}$.

The classical Bloch space $\mathcal{B}$ and little Bloch space $\mathcal{B}_{0}$ correspond to the weight $v(z):=1-|z|^{2}$. Among the many references on these spaces, we mention Zhu [17], for example.

Define the bounded operators $S: \mathcal{B}_{v} \rightarrow H_{v}^{\infty}, S(h)=h^{\prime}$ and $S^{-1}: H_{v}^{\infty} \rightarrow \mathcal{B}_{v},\left(S^{-1} h\right)(z)=$ $\int_{0}^{z} h(\xi) d \xi$. Then $S S^{-1}=i d_{H_{v}^{\infty}}, S^{-1} S=i d_{\mathcal{B}_{v}}$ and $S, S^{-1}$ are isometric onto maps. These operators induce isometries between $H_{v}^{0}$ and $\mathcal{B}_{v, 0}$.

Consider a composition operator $C_{\phi}: \mathcal{B}_{v} \rightarrow \mathcal{B}_{v}$. We can use Theorem 2.3 to find an extremal function $g \in H_{v}^{\infty}$ for the norm of $C_{\phi, \phi^{\prime}}: H_{v}^{\infty} \rightarrow H_{v}^{\infty}$, so that $\left\|C_{\phi}\right\|=\left\|C_{\phi, \phi^{\prime}}\right\|=\left\|S C_{\phi} S^{-1}\right\|=$ $\left\|\left(S C_{\phi} S^{-1}\right) g\right\|_{v}$. Now using that $S$ is an isometry, it follows that $h:=S^{-1}(g) \in \mathcal{B}_{v}$ is an extremal function for the norm of $C_{\phi}: \mathcal{B}_{v} \rightarrow \mathcal{B}_{v}$. Accordingly we get the following extension of Martín [14, Theorem 6].

Corollary 4.1 Every composition operator $C_{\phi}: \mathcal{B}_{v} \rightarrow \mathcal{B}_{v}$ is norm-attaining. 
Proceeding similarly for $C_{\phi}: \mathcal{B}_{v, 0} \rightarrow \mathcal{B}_{v, 0}$ we obtain the following consequence of Corollary 3.4 that is an extension of Martín [14, Theorem 1].

Corollary 4.2 Let $v$ be a polynomial weight $v(z)=\left(1-|z|^{2}\right)^{\alpha}, \alpha>0$. A composition operator $C_{\phi}: \mathcal{B}_{v, 0} \rightarrow \mathcal{B}_{v, 0}$ is norm attaining if and only if there are $b \in \mathbb{D}$ and $\left(z_{n}\right)_{n}$ in $\mathbb{D}$ with $\lim _{n \rightarrow \infty} \phi\left(z_{n}\right)=b$ such that

$$
\sup _{z \in \mathbb{D}} \frac{w(z)\left|\phi^{\prime}(z)\right|}{\tilde{v}(\phi(z))}=\lim _{n \rightarrow \infty} \frac{w\left(z_{n}\right)\left|\phi^{\prime}\left(z_{n}\right)\right|}{\tilde{v}\left(\phi\left(z_{n}\right)\right)} .
$$

\section{References}

[1] K.D. Bierstedt, J. Bonet, A. Galbis, Weighted spaces of holomorphic functions on bounded domains, Michigan Math. J. 40 (1993), 271-297.

[2] K.D. Bierstedt, J. Bonet, J. Taskinen, Associated weights and spaces of holomorphic functions, Studia Math. 127 (1998), 137-168.

[3] J. Bonet, P. Domański, M. Lindström, Essential norm and weak compactness of composition operators on weighted Banach spaces of analytic functions, Canad. Math. Bull. 42, no. 2, (1999), 139-148.

[4] J. Bonet, P. Domański, M. Lindström, J. Taskinen, Composition operators between weighted Banach spaces of analytic functions, J. Austral. Math. Soc. Ser. A 64 (1998), 101-118.

[5] J. Bonet, M. Lindström, E. Wolf, Isometric weighted composition operators on weighted Banach spaces of type $H^{\infty}$, Proc. Amer. Math. Soc. 136 (2008), 4267-4273.

[6] J. Bonet, E. Wolf, A note on weighted spaces of holomorphic functions, Archiv Math. 81 (2003), 650-654.

[7] M.D. Contreras, A.G. Hernández-Díaz, Weighted composition operators in weighted banach spaces of analytic functions, J. Austral. Math. Soc. Ser. A 69 (2000) 41-60.

[8] C. Cowen, B. MacCluer, Composition Operators on Spaces of Analytic Functions, CRC Press, Boca Raton, 1995.

[9] J. Diestel, Geometry of Banach Spaces. Selected Topics, Lecture Notes in Math. vol. 485, Springer, Berlin, 1975.

[10] C. Hammond, On the norm of a composition operator with linear fractional symbol, Acta Sci. Math. (Szeged) 69 (2003), no. 3-4, 813-829.

[11] T. Hosokawa, K. Izuchi, D. Zheng, isolated points and essential components of composition operators on $H^{\infty}$, Proc. Amer. Math. Soc. 130 (2001), 1765-1773.

[12] T. Hosokava, S. Ohno, Topological strusctures of the sets of composition operatorson the Bloch spaces, J. Math. anal. Appl. 303 (2005), 499-508.

[13] W. Lusky, On the isomorphy classes of weighted spaces of harmonic and holomorphic functions, Studia Math. 175 (2006), 19-45.

[14] M. Martín, Norm-attaining composition operators on the Bloch spaces, J. Math. Anal. Appl. 369 (2010), 15-21.

[15] A. Montes-Rodríguez, Weighted composition operators on weighted Banach spaces of analytic functions, J. London Math. Soc. 61 (2000), no. 2, 872-884.

[16] J.H. Shapiro, Composition Operators and Classical Function Theory, Springer, 1993. 
[17] K. Zhu, Operator Theory in Function Spaces. Second Edition, Amer. Math. Soc., 2007.

\section{Authors' Addresses.}

J. Bonet: Instituto Universitario de Matematica Pura y Aplicada IUMPA-UPV, Universidad Politécnica de Valencia, E-46022 Valencia, SPAIN

e-mail: jbonet@mat.upv.es

M. Lindström: Department of Mathematical Sciences, P.O. Box 3000, FIN-90014 University of Oulu, Oulu, FINLAND

e-mail: mikael.lindstrom@oulu.fi

E. Wolf: Institute of Mathematics, University of Paderborn, D-33095 Paderborn, GERMANY. e-mail: lichte@math.uni-paderborn.de 